\title{
Comprehensive identification and analysis of DELLA genes throughout the plant kingdom
}

Pengfei Wang ${ }^{1,2^{*}}$, Qianqian Zhang ${ }^{1}$, Yingchun Chen ${ }^{1}$, Yanxia Zhao ${ }^{1}$, Fengshan Ren ${ }^{1,2}$, Hongmei Shi ${ }^{{ }^{*}}$ and Xinying $\mathrm{Wu}^{1,2^{*}}$

\begin{abstract}
Background: DELLAs play key roles in plant gibberellin signaling pathways and are generally important in plant development and growth. However, DELLAs in many plant taxa have not yet been systematically analyzed.

Results: In our study, we searched for DELLA genes across 58 green plant genomes and found 181 DELLAs. Structure analysis showed some DELLA domains do not contain "D-E-L-L-A" sequences and instead contain similar domains, including DGLLA and DSLLH domains. "VHYNP" motifs in plant DELLAs comprise 23 types of sequences, while some DELLAs did not contain GRAS domains. In grape, we found that the DELLA protein GSVIVT01015465001 contains an F-box domain, while apple DELLA proteins MDP0000220512 and MDP0000403162 contain a WW domain and a BCIP domain, respectively. These DELLAs can be divided into 22 homologous groups and 17 orthologous groups, and 35 paralogous genes were identified. In total, 35 positively selected genes (PSGs) and 121 negatively selected genes (NSGs) were found among DELLAs based on selective pressure analysis, with an average $K_{\mathrm{s}}$ of NSGs that was significantly higher than that of PSGs $(P<0.05)$. Among the paralogous groups, CBI and Fop were significantly positively correlated with $\mathrm{GC}, \mathrm{GC1}, \mathrm{GC2}, \mathrm{GC1} 2$, and $\mathrm{GC} 3$, while CAI was significantly positively correlated with GC, GC1, GC12, and GC. The paralogous groups with $\omega$ values exceeding 1 had significantly higher $K_{\mathrm{a}}$ values. We also found some paralogous groups with $\omega$ values exceeding 1 that differed in their motifs.
\end{abstract}

Conclusions: This study provides helpful insights into the evolution of DELLA genes and offers exciting opportunities for the investigation of DELLA functions in different plants.

Keywords: DELLA, GRAS domain, PSG, NSG, Orthologous, Paralogous

\section{Background}

The development of a plant is an orderly process that starts from germination and continues to maturity, and it is modulated by environmental conditions and internal phytohormones, such as abscisic acid, cytokinins, ethylene, auxins, and gibberellin (GA). GA can modulate seed germination and stem and flower development

\footnotetext{
*Correspondence: fengqiaoyouzi@126.com; hm.shi@163.com; echomoon0622@163.com

'Shandong Academy of Grape, Shandong engineering research center for Grape cultivation and deep-processing, Jinan 250100, China

Full list of author information is available at the end of the article
}

processes, among other developmental processes [1-4]. Arabidopsis thaliana GA-deficient mutant ga1-3 contains greatly reduced levels of endogenous GA; this mutant is defective in germination, impaired in the development of its flowers, and retarded in its vegetative growth [5-7]. DELLA proteins play a key role in the plant GA signaling pathway. DELLA proteins can regulate gene transcription, restrict plant development, and repress GA signaling $[8,9]$.

The most obvious feature of DELLA proteins is the Nterminal DELLA domain. Some DELLA domains (Pfam

(c) The Author(s). 2020 Open Access This article is licensed under a Creative Commons Attribution 4.0 International License, which permits use, sharing, adaptation, distribution and reproduction in any medium or format, as long as you give appropriate credit to the original author(s) and the source, provide a link to the Creative Commons licence, and indicate if changes were made. The images or other third party material in this article are included in the article's Creative Commons licence, unless indicated otherwise in a credit line to the material. If material is not included in the article's Creative Commons licence and your intended use is not permitted by statutory regulation or exceeds the permitted use, you will need to obtain permission directly from the copyright holder. To view a copy of this licence, visit http://creativecommons.org/licenses/by/4.0/ The Creative Commons Public Domain Dedication waiver (http://creativecommons.org/publicdomain/zero/1.0/) applies to the data made available in this article, unless otherwise stated in a credit line to the data. 
ID: PF12041) previously identified were found to contain "D-E-L-L-A" amino acid sequences [10]. DELLA proteins belong to the GRAS gene family and are a type of plant-specific nuclear protein. However, since DELLA proteins do not contain a canonical DNA-binding domain, they may interact with other transcription factors and then regulate the target genes. DELLA proteins can interact with many transcription factors, including ABA INSE NSITIVE 3 (ABI3), ABI5, AUXIN RESPONSE FACTOR 6 (ARF6), PHYTOCHROME INTERACTING FACTORs (PIFs), BRASSINAZOLE-RESISTANT 1 (BZR1), ETHYLE NE-INSENSITIVE 3 (EIN3), JASMONATE-ZIMDOMAIN PROTEINs (JAZs), DWARF 14 (D14), and FLOWERING LOCUS C (FLC), as well as brassinosteroid (BR) signaling, which are involved in multiple phytohormone signaling pathways, thus participating in complex crosstalk among plant hormones [11-17].

In $A$. thaliana, five DELLA proteins were identified genome-wide: RGALIKE1 (RGL1), RGL2, RGL3, GA INSENSITIVE (GAI), and REPRESSOR OF GA1-3 (RGA). RGA, RGL1, and RGL2 have been shown to regulate floral development $[18,19]$. RGL2 can also repress seed germination [20]. RGL3 represses testa rupture during seed germination [21]. RGA and GAI can repress vegetative growth $[22,23]$. In maize, two DELLA proteins, DWARF8 (D8) and DWARF9 (D9), were identified [24], while the DELLA proteins REDUCED HEIG HT-1 (RHT-1) [25], SLENDER RICE1 (SLR1) [26], PROCERA [27], and VvGAI1 [28] were identified in wheat, rice, tomato, and grapevine, respectively. Additionally, four MeDELLAs have been identified in cassava [29].

DELLAs are important in plant development and growth and can interact with TCP transcript factors to affect the development of the inflorescence shoot apex and thus control plant height [29]. Leaf senescence can also be regulated by DELLAs [30]. DELLA proteins can regulate plant reproductive organ size, affect fertilization, and promote fruit growth [31]. Furthermore, DELLA proteins can promote the development of nodules and formation of infection threads during root nodule symbiosis; they can also control arbuscular mycorrhizal symbiosis in plants [32-34].

DELLAs play important roles in several aspects of plant development and growth that are influenced by environmental cues [35-40]. As such, DELLA proteins can improve the survival of plants during adversity $[35,36]$. Researchers have concluded that DELLA proteins can integrate environmental signals, so that plants can alter their growth in response to the surrounding environment. DELLA proteins are also involved in responses to biotic stress [37, 41]. DELLA proteins can improve the tolerance of necrotrophs by potentiating jasmonate signaling [41]. Additionally, DELLA proteins can suppress laccase-like multicopper oxidase activity in response to herbivory and regulate glucosinolate levels [42].

DELLAs in many plant taxa have not yet been systematically analyzed. In this study, we searched for DELLA genes across 58 plant genomes and found a total of 181 DELLAs. This work provides helpful insights into the evolution of DELLA genes and offers important opportunities for the investigation of DELLA functions in different plant species, building upon previous research conducted on DELLA genes in many species. We performed a comprehensive analysis of DELLA genes throughout the plant kingdom, including analyses of their protein sequences, molecular signatures of selection, and codon usage patterns. We also analyzed the gene tree of these DELLAs using phylogenetic methods and identified orthologs, including PSGs, NSGs, and paralogs in 58 plant species. In addition, we performed other molecular evolution analyses of orthologs, paralogs, PSGs and NSGs, respectively, and relatively new and old DELLA genes, as well as improving knowledge about the VHYNP domain of DELLA proteins.

\section{Results}

\section{Validation and extension of DELLA gene databases}

Using the HMMER3 $\mathrm{hmm}$ search command, we identified 181 DELLAs from the 66 green plant genomes examined. Among the genomes of all Chlorophyta and the moss Physcomitrella patens, DELLA genes were not identified using the method described. Accordingly, DELLA genes were identified in 58 species in this study. To validate our searching method, we compared our list of DELLA genes with those reported in the literature. Our search successfully found all five DELLAs previously reported in $A$. thaliana [43], one reported in tomato [44], four reported in Populus trichocarpa [44], two reported in maize [24], one reported in rice [45], and two reported in Selaginella moellendorffii [29]. A previous study identified four DELLA genes in soybean [44], and two additional DELLA genes were identified in this species. Three DELLA genes were previously identified in the Medicago truncatula Mt3.5 genome database, and we only identified two DELLA genes in the newer $M$. truncatula Mt4.0 genome database (https://phytozome. jgi.doe.gov/pz/portal.html\#!linfo?alias=Org_Mtruncatula). Furthermore, four DELLA genes were previously identified in cassava [46], while we only identified three DELLA genes in cassava based on a pfam domain hidden Markov model (HMM) database (Table S1).

Among bryophytes, we identified two DELLA genes in Sphagnum fallax and one in Marchantia polymorpha. In Amborella trichopoda, which belongs to the order Amborellales, we identified three DELLA genes. Among Gymnosperms, we identified two DELLA genes in Ginkgo biloba, two in Pinus pinaster, and one in Picea 
abies. Interestingly, in Picea abies, there was a protein with a sequence similar (identity $>80 \%$ ) to that of a Pinus pinaster DELLA gene (sp_v3.0_unigene6469); however, the protein was not found by our identification strategy, since it does not contain a DELLA domain. Accordingly, it is not strictly considered to be a DELLA gene. We found that the apple genome examined contains the most DELLA genes, i.e., 14 in total. Most monocotyledons were found to contain only one DELLA gene. The longest DELLA sequence was that of GSVIVT01015465001 in grape, while the shortest was MDP0000855334 in apple (Table S1). The number of DELLA genes identified was neither correlated with the number of whole-genome duplications (WGDs) nor genome size, indicating that WGD events did not directly affect the number of DELLA genes.

We also determined the conserved motifs and domains of these plant DELLA proteins. Using MEME, we identified 10 conserved motifs (Fig. S1). Motif7 partly overlaps with most DELLA domains, excluding Sphfalx0442s0002.1 in Sphagnum fallax, 122,441 in Selaginella moellendorffii, GSVIVT01030735001 in grapevine, MDP0000855334, MDP0000298557, MDP0000220512, and MDP0000192154 in apple, Spipo15G0028300 in Spirodela polyrhiza, and GSMUA_Achr9T13490_001 in Musa acuminata (Fig. S2). Most copies of motif7 started with "MDELLA" or "YDELLA" amino acid sequences. However, nine DELLA proteins do not contain motif7, and their DELLA domains differed more from other DELLA proteins and were shorter as well, such as apple DELLA protein MDP0000855334. Moreover, 67 DELLA domains do not contain "DELLA" sequences and instead contained domains, such as DGLLA, DSLLH, and other peptide strings of the "DXLLX" form.
Additionally, some DELLA domains contain neither "DELLA" sequences nor the "DXLLX" form. For example, in Sphagnum fallax, DELLA Sphfalx0198s0025.1 does not contain a typical "DELLA" or "DXLLX" five-peptide structure, but instead contains an "RNCNE" structure (Fig. 1).

Most plant DELLA proteins contain GRAS domains. We found most DELLA proteins contain one DELLA domain and one GRAS domain. Some only contain a DELLA domain; some contain one DELLA domain and two GRAS domains; some contain one DELLA domain and three GRAS domains, and others contain two DELLA domains and one GRAS domain. Grape DELLA protein GSVIVT01015465001 contains one DELLA domain, one F-box domain, and one GRAS domain. Apple DELLA protein MDP0000220512 contains one DELLA domain and one WW domain. Apple DELLA protein MDP0000403162 contains one DELLA domain and one BCIP domain (Table S1).

The VHYNP motif of DELLA was identified based on its "VHYNP" sequences. VHYNP motifs were reported in rice as part of the "TVHYNP" domain [47]. VHYNP motifs of the DELLA protein RGA are needed for GID1 interactions in A. thaliana [48]. Previous studies have only reported VHYNP motifs in rice, maize, and Arabidopsis $[48,49]$, and Cassani et al. even referred to it as a putative VHYNP motif [49]. However, VHYNP motifs were not reported in other plants, and there was not a related pfam domain HMM in the pfam database. Motif10 partly overlaps with VHYNP motifs (Fig. S2). Because there is no VHYNP pfam domain HMM, we identified the VHYNP motifs of plant DELLA proteins based on identification of motif10 in plant DELLA

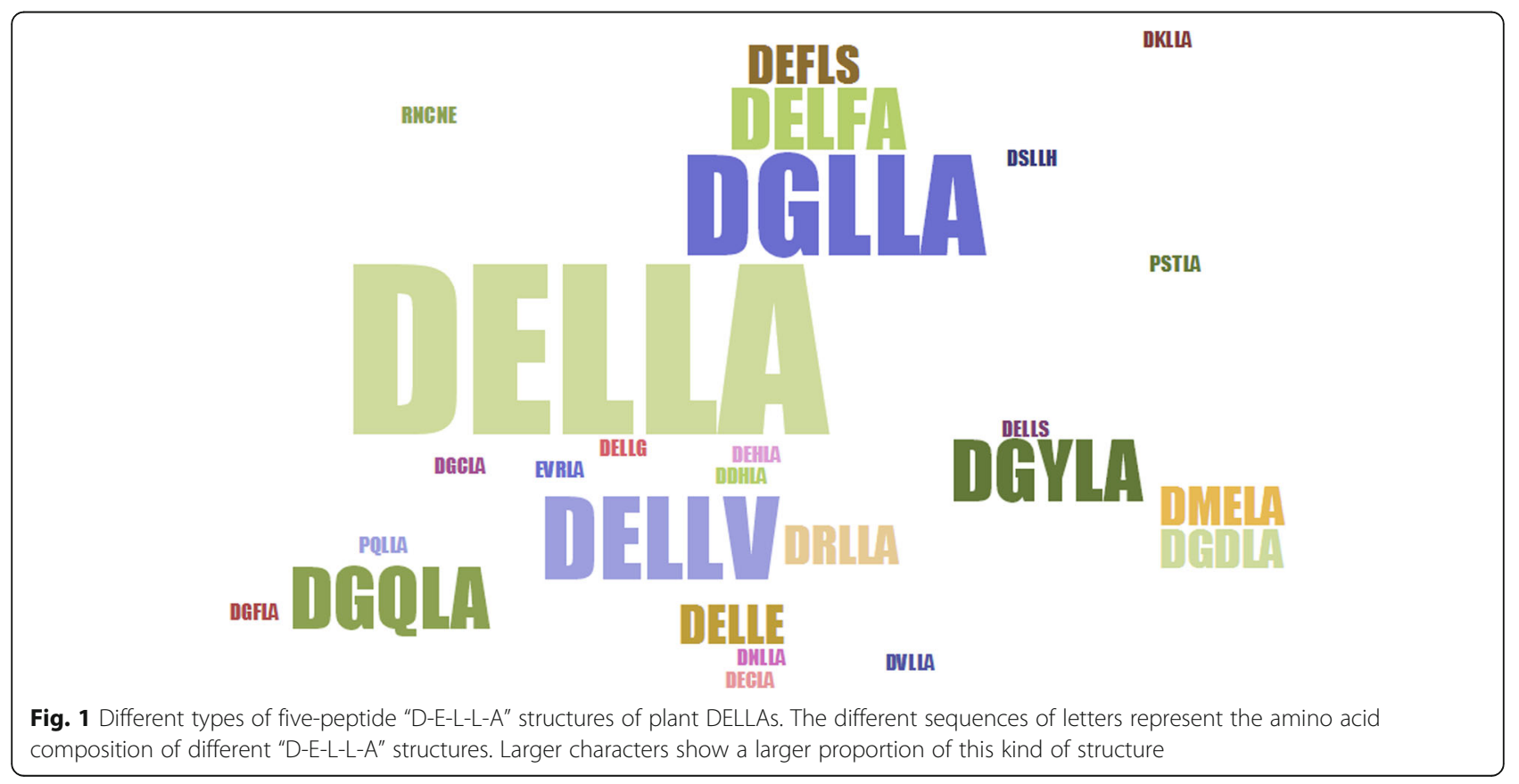


proteins. Through detecting these instances of motif10, we found five DELLA proteins that did not contain a motif10/VHYNP motif. "VHYNP" motifs comprise 23 kinds of sequences, including "VHYNP," "VHVDP," and "VFYNP" sequences (Fig. 2).

\section{Gene tree analysis of DELLA sequences}

Because the ancestral DELLA gene sequence is unknown, and the extant gene closest to the ancestral gene sequence is unknown, we constructed an unrooted gene tree without an outgroup. Our gene tree analysis included all DELLA proteins identified in our search. We used the entire prepropeptide in our gene tree analysis. A previous study showed that plant DELLAs can be divided into three large groups. Here, our gene tree could be divided into 22 homologous groups based on bootstrap value $(>700)$, with some orphans, such as the Amaranthus hypochondriacus DELLA, AHYPO_001751RA (Fig. 3). A previous study showed a group that contained genes from different species with a bootstrap value $>700$ could be considered an orthologous group [50]. Here, paralogous groups only contained genes from the same species with a bootstrap value of at least 700 and could not be included in any other group with a bootstrap value of at least 700. Accordingly, the DELLAs from the same species in the same homologous group were considered paralogs, and the DELLAs from different species in the same homologous group were considered orthologs. In other words, if a homologous group contained DELLAs from different species, it could also be called an orthologous group. Based on bootstrap values, we identified 17 homologous groups, including groups 1, 2, 3, 5, 6, 7, 9, 12, and 13-21. (Fig. 3). Within an orthologous group, the genes included are orthologs of each other. Group 1 contained the most DELLAs and species (i.e., 30 species). DELLAs of the nonspermatophyte plants, i.e., bryophytes and Selaginella, only belonged to group 1 . DELLAs from 10 monocotyledons (all gramineous species) are independently grouped into group 3. Zostera marina and Spirodela polyrhiza are aquatic angiosperms. One $Z$. marina DELLA (Zosma208g00370).1 was grouped into group 1, and two other Z. marina DELLAs were grouped into group 4. One $S$. polyrhiza DELLA (Spipo0G0167100) was grouped into group 1, while another S. polyrhiza DELLA (Spipo15G0028300) was grouped into group 2, and yet another (Spipo15G0027800) was an orphan. DELLAs of nine types of cruciferous plants, including Arabidopsis, were independently divided into groups 9 and 15 . The result is in agreement with a previous study, in which

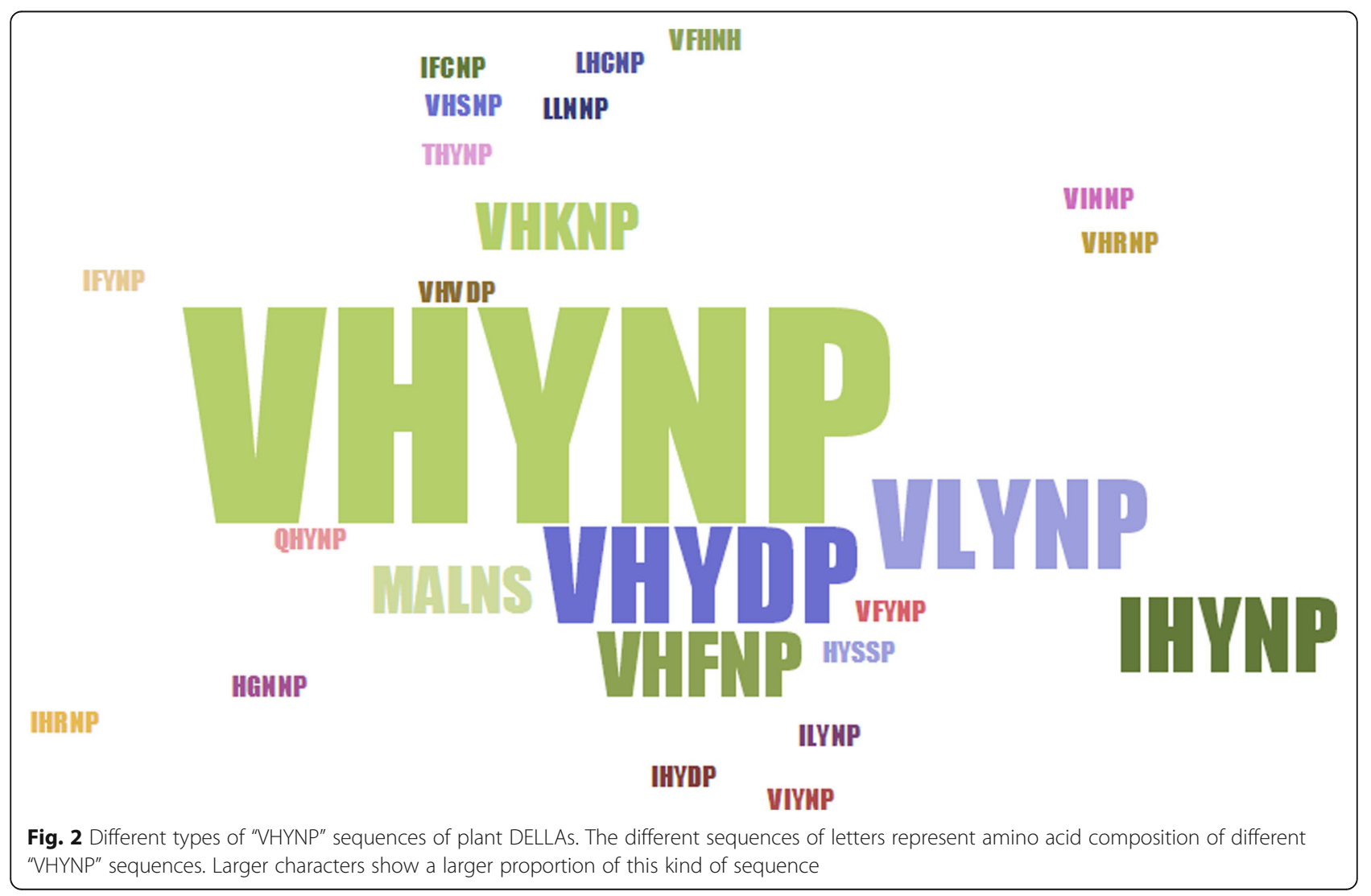




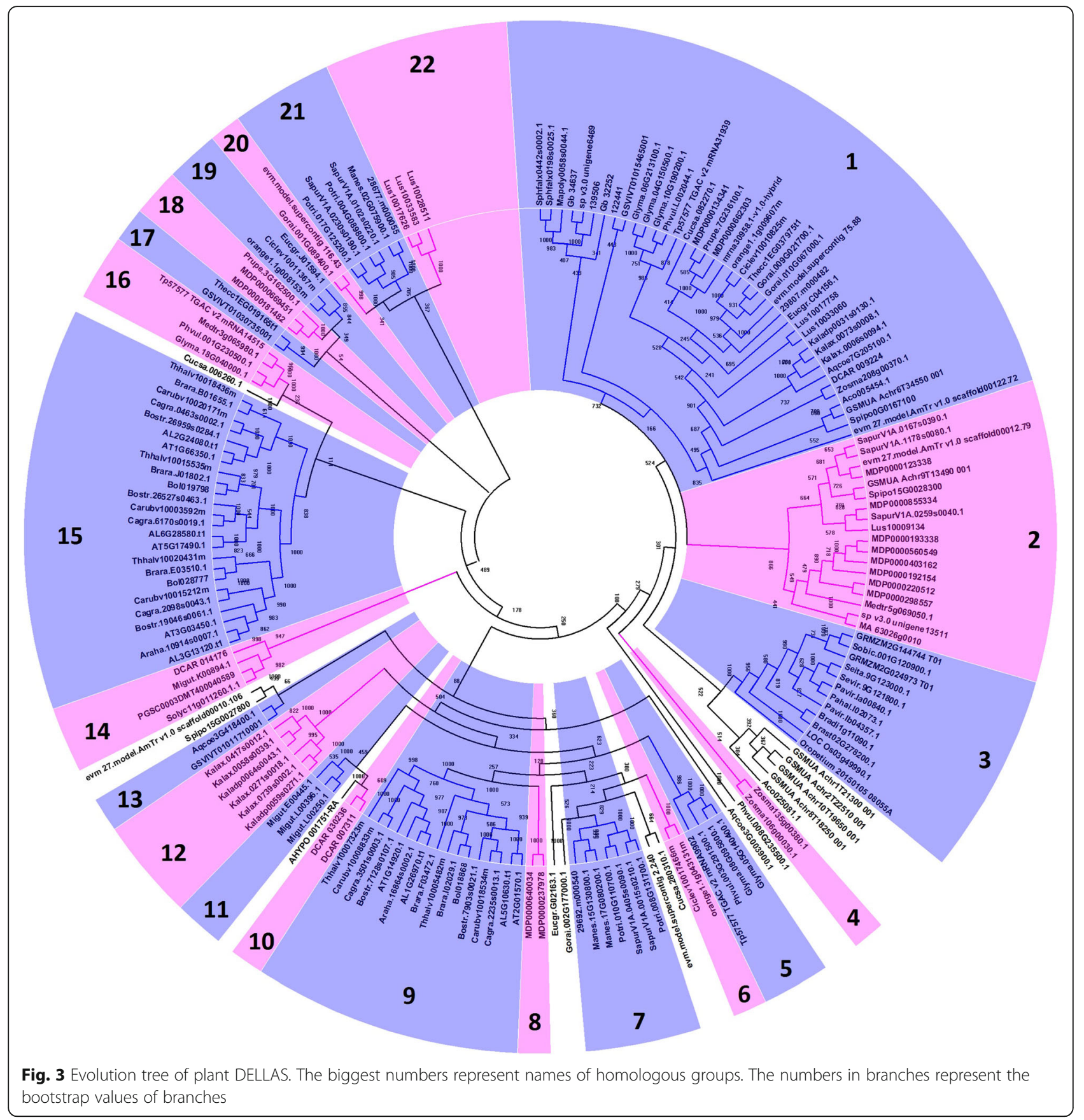

Arabidopsis DELLA GAI (AT1G14920.1) and RGA (AT2G01570.1) were grouped into group 9, while RGL1 (AT1G66350.1), RGL2 (AT3G03450.1), and RGL3 (AT5G17490.1) were grouped into group 15 [44]. Cruciferous Arabidopsis thaliana, Capsella grandiflora, Boechera stricta, Arabidopsis lyrata, and Capsella rubella all contain five DELLAs, and all have two DELLAs in group 9 and three DELLAs in group 15. However, A. halleri only contained two DELLAs, one in group 9 and one in group 15. Group 16 only contains DELLAs of leguminous plants. Group 22 only contains the DELLAs of Linum usitatissimum. Most apple DELLAs were divided into groups 1 and 2, and group 12 only contained Kalanchoe DELLAs (Fig. 3).

Paralogs were derived from duplication events. Non-paralogous (non-duplication) DELLA genes were considered singleton DELLA genes in our study. Accordingly, we identified 101 duplicate DELLA genes. Grape DELLA genes were all singletons, while all apple DELLA genes were duplicates. Most soybean 
DELLA genes were duplicates, but one was a singleton.

\section{Molecular evolution analysis of orthologous groups, PSGs, NSGs, and new and old DELLA genes}

For each orthologous group, $K_{\mathrm{a}}, K_{\mathrm{s}}$, and $K_{\mathrm{a}} / K_{\mathrm{s}}(\omega)$ values were calculated (Table 1 ), where $K_{\mathrm{a}}$ and $K_{\mathrm{s}}$ represent rates of molecular evolution. Overall, $K_{\mathrm{a}}$ ranged from 0.0122 to 8.8684 , with a mean of 1.48 (Table 1 ). The $K_{\mathrm{s}}$ estimates ranged from 0.0164 in group 6 to 12.8 in group 1, with a mean of 3.160 (Table 1). These results showed that the divergence time of group 1 was most ancient, while that of group 6 was the most recent. The $K_{\mathrm{a}} / K_{\mathrm{s}}$ estimates ranged from 0.0389 to 3.966 , with a mean of 0.748 (Table 1). Previous studies have shown that genes could be classified into PSGs and NSGs, which have undergone purifying selection and neutral genes [51]. The identification of PSGs and NSGs is based on the separate $K_{\mathrm{a}} / K_{\mathrm{s}}$ values of each orthologous group. If the $\omega$ vales of an orthologous group exceed 1, the genes are considered PSGs $[52,53]$. If the $\omega$ value of an orthologous group is less than 1, the genes are considered NSGs $[52,53]$. In our study, $\omega$ values of four orthologous groups were found to be greater than 1, while the others were less than 1 . In this study, 35 PSGs and 121 NSGs were found among all the DELLA genes (Table S2). We found that $K_{\mathrm{a}} / K_{\mathrm{s}}$ increases gradually as $K_{\mathrm{s}}$ increases in the NSGs $(r=0.5) . K_{\mathrm{a}} / K_{\mathrm{s}}$ was negatively correlated with $K_{\mathrm{s}}(r=-0.65)$ among the PSGs. As shown in Table 2, the average $K_{\mathrm{s}}$ of NSGs is 3.9, which was significantly higher than the average $K_{\mathrm{s}}$ of PSGs

Table $1 \mathrm{Ka}, \mathrm{Ks}$ and $\mathrm{Ka} / \mathrm{Ks}$ value of all homologous groups

\begin{tabular}{llll}
\hline Group name & $\mathrm{Ka}$ & $\mathrm{Ks}$ & $\mathrm{Ka} / \mathrm{Ks}$ \\
\hline Group1 & 8.8684 & 12.8116 & 0.69222 \\
Group2 & 8.375 & 9.9533 & 0.84143 \\
Group3 & 0.2495 & 2.8828 & 0.08654 \\
Group5 & 0.966 & 0.4598 & 2.10117 \\
Group6 & 0.0122 & 0.0164 & 0.74373 \\
Group7 & 0.3385 & 2.1128 & 0.16021 \\
Group9 & 0.6163 & 3.6567 & 0.16855 \\
Group12 & 0.0879 & 0.504 & 0.17445 \\
Group13 & 0.7823 & 0.4619 & 1.69379 \\
Group14 & 0.4106 & 6.8241 & 0.06017 \\
Group15 & 2.6585 & 1.8314 & 1.4516 \\
Group16 & 0.3155 & 2.4392 & 0.12935 \\
Group17 & 0.3331 & 3.0723 & 0.10842 \\
Group18 & 0.1154 & 0.775 & 0.14895 \\
Group19 & 0.6071 & 0.1531 & 3.96623 \\
Group20 & 0.1427 & 3.6662 & 0.03891 \\
Group21 & 0.3323 & 2.2607 & 0.14701 \\
\hline
\end{tabular}

Table 2 Various indexes of PSG and NSG

\begin{tabular}{llll}
\hline & NSG & PSG \\
\hline Ks & $3.921 \pm 3.625$ & $0.726 \pm 0.65$ \\
Ka & $1.553 \pm 0.766$ & $1.253 \pm 0.821$ \\
CAI & $0.238 \pm 0.03$ & $0.22 \pm 0.02$ \\
CBI & $0.1 \pm 0.09$ & $0.08 \pm 0.05$ \\
Fop & $0.476 \pm 0.05$ & $0.477 \pm 0.03$ \\
Exon length & $1533 \pm 428$ & $1620 \pm 126$ \\
GC & $0.546 \pm 0.077$ & $0.515 \pm 0.047$ & $*$ \\
GC3 & $0.60 \pm 0.17$ & $50.4 \pm 0.11$ & $*$ \\
\hline
\end{tabular}

"*" represents significant difference $(P<0.05)$

(0.72; $P<0.05)$. We also calculated the codon preference index and found that the average codon adaption index (CAI) and GC and GC3 contents of NSGs were significantly higher than those of PSGs (Table 2).

A previous study determined that the genes in a genome differ in age in the sense that they have identifiable orthologs across a diverse range of species spanning vast evolutionary distances. Some genes are younger, in the sense that orthologs are identifiable only in closely related species [54]. Accordingly, we considered genes in orthologous groups to be old DELLA genes, while the others were considered new DELLA genes. The new genes included two DELLA genes in Z. marina, three in Mimulus guttatus, two in apple, two in Daucus carota, and three in Linum usitatissimum. The Fop, CAI, and CBI values of both new and old DELLA genes were positively correlated with GC content. The CAI values of new DELLA genes were positively correlated with exon length. CBI and Fop of old DELLA genes were positively correlated with exon length $(r>0.1)$. The GC12 content of both new and old DELLA genes was significantly positively correlated with GC3 content $(r>0.75, P<0.01$; Table 3).

\section{Identification and molecular evolution analysis of paralogous groups}

A previous study considered a group containing genes from the same species with a bootstrap value $>700$ to be a paralogous group within a gene tree [50], regardless of whether or not the group is already included in an orthologous group. In group 1, we identified nine paralogous groups, including a positively selected paralogous group of Selaginella moellendorffii DELLA genes. In group 2, we identified two paralogous groups, including a positively selected paralogous group of apple DELLA genes. In homologous group 3, we identified two paralogous groups. Group 4 is also a paralogous group that only includes two Z. marina DELLA genes. In group 5, we identified one paralogous group, including two soybean DELLA genes. In group 7, we identified one paralogous group, including two Manihot esculenta 
Table 3 Correlation analysis among various indexes of new and old genes

\begin{tabular}{llll}
\hline New gene & & & \\
\hline GC & CAl & CBI & Fop \\
exon length & 0.27403 & $0.61746^{*}$ & $0.59282^{*}$ \\
GC1 & 0.10988 & -0.02117 & 0.00402 \\
GC2 & 0.1788 & $0.47283^{*}$ & $0.43599^{*}$ \\
GC12 & 0.1375 & $0.47316^{*}$ & $0.46518^{*}$ \\
GC3 & 0.16778 & $0.49214^{*}$ & $0.46658^{*}$ \\
Old gene & 0.32925 & $0.66561^{*}$ & $0.6429^{*}$ \\
& & & \\
GC & CAI & CBI & Fop \\
exon length & $0.49037^{*}$ & $0.78934^{*}$ & $0.77408^{*}$ \\
GC1 & 0.04337 & $0.16199^{*}$ & 0.15033 \\
GC2 & $0.465^{*}$ & $0.66137^{*}$ & $0.63796^{*}$ \\
GC12 & $0.18153^{*}$ & $0.36352^{*}$ & $0.36707^{*}$ \\
GC3 & $0.38737^{*}$ & $0.60739^{*}$ & $0.59468^{*}$ \\
\hline u*" represents significant & $0.49707^{*}$ & $0.80981^{*}$ & $0.79306^{*}$ \\
\hline
\end{tabular}

"*" represents significant correlation $(\mathrm{P}<0.05)$

DELLA genes. Group 8 is also a paralogous group that only includes two apple DELLA genes. In group 9, we identified seven paralogous groups, including a positively selected paralogous group of Arabidopsis lyrate DELLA genes. Groups 10 and 11 are also paralogous groups. In group 12, we identified two paralogous groups. In group 15 , we identified eight paralogous groups, including a positive paralogous group of $A$. lyrate DELLA genes. In group 21, we identified two paralogous groups, and in orthologous group 22, we identified one paralogous group. Group 22 is also a paralogous group consisting of only three apple genes (Fig. 3).

We also compared the average $K_{\mathrm{a}}, K_{\mathrm{s}}$, and $\omega$ values of paralogous groups with those of orthologous groups. The average $\omega$ value of paralogous groups was 0.46 , and the average $\omega$ value of orthologous groups was 0.7 . The average $K_{\mathrm{s}}$ value of paralogous groups was 0.8 , and the average $K_{\mathrm{s}}$ value of orthologous groups was 3.16. The average $K_{\mathrm{a}}$ value of paralogous groups was 0.36 , and the average $K_{\mathrm{a}}$ of orthologous groups was 1.48. In both the orthologous and paralogous groups, the $K_{\mathrm{s}}$ values were negatively correlated with $\omega$ values, while the $\omega$ values were negatively correlated with $K_{\mathrm{s}}$ values and positively correlated with $K_{\mathrm{a}}$ values both in orthologous groups and paralogous groups $(r>0.1)$. In the paralogous groups, CBI and Fop were significantly positively correlated with GC, GC1, GC2, GC12, and GC3, and CAI was significantly positively correlated with $\mathrm{GC}$, GC1, GC12, and GC (Table 4). The paralogous groups for which $\omega$ was greater than 1 had
Table 4 Correlation analysis among various indexes of paralogous

\begin{tabular}{llll}
\hline & CAl & CBI & Fop \\
\hline GC & $0.52825^{*}$ & $0.76662^{*}$ & $0.75238^{*}$ \\
GC1 & $0.42804^{*}$ & $0.53809^{*}$ & $0.50243^{*}$ \\
GC2 & 0.10389 & $0.26008^{*}$ & $0.26722^{*}$ \\
GC12 & $0.33572^{*}$ & $0.50442^{*}$ & $0.4865^{*}$ \\
GC3 & $0.55326^{*}$ & $0.79438^{*}$ & $0.78327^{*}$ \\
exon length & -0.00552 & 0.14136 & $0.12601^{*}$ \\
\hline u*" represents significant correlation $(P<0.05)$ & &
\end{tabular}

significantly higher average $K_{\mathrm{a}}$ values. Accordingly, they had faster rates of amino acid substitution and evolution. We also analyzed the motifs of paralogous groups for which $\omega$ was greater than 1 . We found some paralogous groups for which $\omega$ was greater than 1 that differed with respect to their motifs; for example, one group, containing DELLAs 122,441 and 139,506 , with the latter containing motif7. However, 122,441 did not contain motif7 (Fig. S1); this may have been caused by positive selection leading to a new gene function [55].

\section{Discussion}

We identified DELLA genes throughout the plant kingdom but were unable to find DELLA genes from green algae, demonstrating that DELLAs were derived in the lineage leading to land plants. Previous studies have failed to report DELLAs in bryophyte genomes; however, we found DELLAs in Marchantia polymorpha and Sphagnum fallax, although we did not find DELLAs in Physcomitrella patens. Accordingly, it is unclear which bryophytes contain DELLA genes, and this remains to be studied in further detail. Chlorophyta, which live in water, do not have DELLAs. However, Z. marina and $S$. polyrhiza, which are aquatic vascular plants, do have DELLAs. Z. marina and S. polyrhiza are angiosperms, which demonstrate that aquatic vascular plants have not lost DELLAs.

The expansion of many other reported gene families has also been related to WGD events [46]. We found that the number of DELLA genes was not related to genome size or the number of WGD events. Accordingly, we deduced that WGD events have not led to the expansion of the DELLA gene family. Gene family contraction can also be related to gene loss events [56]. In Picea abies, there is a protein that is highly similar (identity > 80\%) to a DELLA gene in Pinus pinaster (sp_v3.0_unigene6469); however, the protein was not identified by our identification strategy. Because it does not contain a DELLA domain, it is not considered to be a DELLA gene. This may therefore be considered a loss event of a DELLA gene in Picea abies. Similarly, most cruciferous 
plants contained five DELLA genes, but a few contained two DELLA genes, which may also be explained by one or more loss events.

A previous study strictly described the "D-E-L-L-A" structure of the five-peptide ELLA domain [57]. However, we found many related five-peptide structures that had diversified and were not "DELLA" specifically, including "DGLLA," "DSLLH," and other peptide strings of the "DXLLX" form. Additionally, some DELLA domains contain neither "DELLA" sequences nor sequences in the "DXLLX" form. This shows the variability of DELLA domain structure. In the fern Selaginella, one DELLA gene had the five-peptide "D-E-L-LA" structure. Similarly, most terrestrial monocotyledons contain DELLA genes with a five-peptide "D-E-L-L-A" structure. Z. marina, an aquatic monocotyledon, has DELLA genes that do not contain the typical fivepeptide "D-E-L-L-A" structure. A previous study reported that the DELLA family is a subfamily of the GRAS superfamily [58]. However, we found that many plant DELLAs do not contain the GRAS domain, such as the apple DELLAs MDP0000192154 and MDP0000220512. A previous study reported that early plants (e.g., Spirogloea muscicola) gained a GRAS domain from microorganisms in the soil [59]. While the origins of the DELLA domain remain unknown, we were able to find DELLA domains in some bryophytes.
Previous studies have also reported that the DELLA is a type of GRAS protein, because those reported DELLAs were found to contain GRAS domains. Here, we found some DELLAs without GRAS domains. This raises the question of whether a DELLA protein is a GRAS protein. Here, we found one DELLA that also contained a fused F-box domain (grape DELLA, GSVI VT01015465001), and one that contained a fused WW domain (apple DELLA, MDP0000220512). Some Musa acuminate DELLAs contained three repeat GRAS domains, and it appears that a DELLA domain loss event happened in Picea abies (Fig. 4). A previous study also reported some details about the VHYNP motif in DELLA genes [57]. Here, we found most plant DELLAs contain the VHYNP motif, and these related motifs are also diverse, including five-peptide "VHYNP," "VHVDP," and "VFYNP" structures.

Our gene tree analysis revealed results that contrasted with a previous study that divided DELLAs into three groups based on approximately one dozen species [44]. Here, we divided DELLAs into 22 groups, with some orphan groups, based on many more species. In other words, we found as many as 22 orthologous groups. Orthologs often share similar functions [60]. Accordingly, we conclude that DELLA genes may have become very functionally diversified throughout the plant kingdom. Accordingly, many functions of the plant DELLA

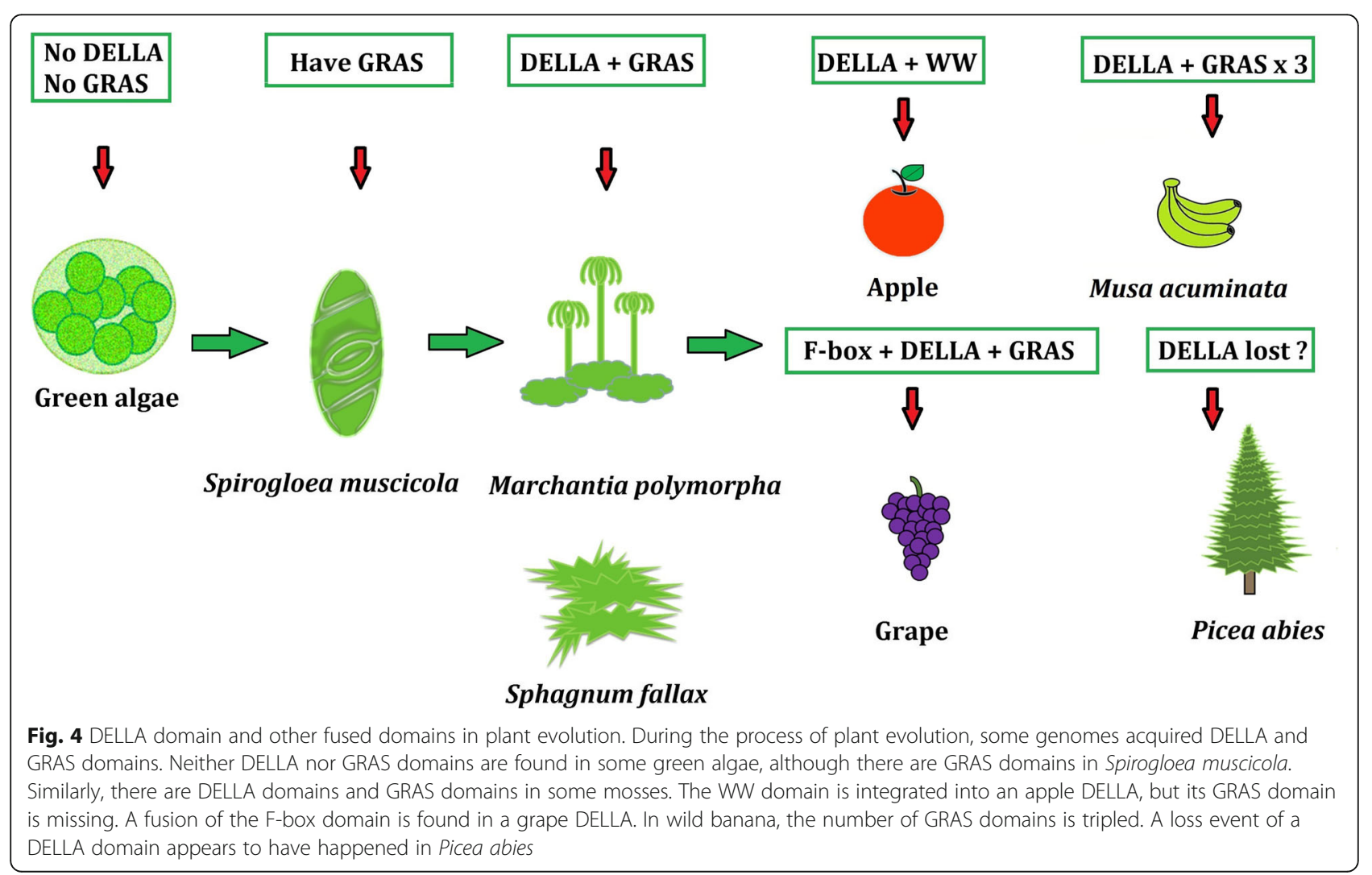


genes remain unknown. A previous study reported that DELLA genes from cruciferous plants could be divided into two groups, which is consistent with the results of our study. However, our finding is that DELLA genes from crucifers could be divided into two independent groups without any other plants. We found that group 1 contains most types of plants, other than those in the families Cruciferae and Gramineae. The Gramineae DELLA genes could be divided into an independent group. Only group 1 contained bryophyte DELLA genes, showing that group 1 may correspond to the ancestral gene family members. Additionally, one aquatic Z. marina DELLA was assigned to group 1 , showing that this gene copy may be derived from the ancestral copy of the many other DELLAs.

Among these orthologous groups, some PSGs were also identified. PSGs were deduced to be under positive selection throughout evolutionary history, with positive selection being related to the adaptation to environments of these species [61-63]. In the plant kingdom, many DELLA genes appear to be involved in tolerance of various abiotic stresses and a range of developmental processes [29-32, 35, 36]. Orthologous groups contain PSGs that may exhibit functional divergence or the evolution of new DELLA gene functions [55, 61-63]. Accordingly, the evolution of plant DELLA genes appears to have been closely related to environmental adaptation and appears to have driven diverse functions underlying adaptation to the environment. Two Z. marina DELLA genes were independently included into one orthologous group (and were also considered to be two new genes); accordingly, the two DELLA genes may pertain to adaptation to aquatic environments. This suggests that various plant DELLAs may still have many unknown functions awaiting discovery. We also found the molecular evolutionary rate of DELLA orthologs to be faster than that of paralogs. Accordingly, the emergence of new species is likely to be associated with new functions among DELLA genes. Species diversification may be a driving force for the emergence of DELLA genes with different functions or multiple functions, as adaptation differs among species under different environments or with different developmental patterns.

The average $K_{\mathrm{s}}$ values of NSGs were significantly higher than those of PSGs, indicating that the average molecular evolutionary rate of DELLA NSGs was higher [64]. The average $K_{\mathrm{s}}$ of PSGs being significantly lower also shows that the average divergence time of orthologous PSGs occurred later [50, 53, 61, 65]. This also shows that the DELLA family may have primarily undergone negative selection at first, with positive selection occurring later. PSGs among the orthologous groups were under positive selection. Previous studies have shown that positive selection may be involved in gene function losses, adaptive evolution, and pseudogenization [62-64]. Positive selection could also lead to new gene functions [55]. For example, homologous group 13 is an orthologous group that contains PSGs, with some PSGs in group 13 containing different motifs. Moreover, different motifs may be related to different functions; in other words, the functions of some PSGs may be different between orthologs. Accordingly, we presumed that recent divergence (sequence divergence or nucleotide substitution) may be more related to the function divergence of DELLAs.

Within the paralogous groups, CBI and Fop were significantly positively correlated with GC3, GC2, GC1, $\mathrm{GC}$, and GC12. Within paralogous groups, CAI was also significantly positively correlated with GC12, GC, GC1, and GC. This showed that paralogs underwent mutation selection [66, 67]. The paralogous groups for which $\omega$ exceeds 1 have significantly higher $K_{\mathrm{a}}$ values, indicating that their rate of amino acid substitution is higher. Some paralogous groups for which $\omega$ exceeds 1 differed in their motifs, and different motifs may be related to different functions. This shows that positive selection may have led to new functions of DELLA genes [55]. In future work, we aspire to determine whether PSGs and paralogs with different motifs have functions that differ from their homologs based on the results of this study. We could also detect whether DELLAs with specific domains correspond to specific functions, and this work is poised to discover additional new functions of DELLA proteins.

\section{Conclusions}

We searched for DELLA genes across 58 green plant genomes and found a total of 181 DELLAs. We analyzed the gene tree of these DELLAs using phylogenetic methods and identified orthologs and paralogs in 58 plant species. We also performed molecular evolution analyses of PSGs, NSGs, and new and old DELLA genes, respectively, as well as improving the knowledge about VHYNP domain of DELLA proteins. Our study provides helpful insights into the evolution of DELLA genes and offers substantial opportunities for the investigation of DELLA functions across plant species.

\section{Methods}

Data collection and identification of plant DELLA genes

In total, 66 plant genomes were obtained for analysis, including 63 green plant genomes from the Phytozome 12.1.6 database (https://phytozome.jgi.doe.gov/pz/portal. html) and the gymnosperm genomes of Ginkgo biloba [68] from GigaDB (http://gigadb.org/), Pinus pinaster from SustainPine DB (http://www.scbi.uma.es/pindb/), and Picea abies from the Congenie.org database (http:// congenie.org). Based on the above data, we used the 
HMMER3 hmm search command to identify all possible DELLA protein candidates in the plant genome database [69]. We used the online software SMART (http://smart. embl-heidelberg.de/) to identify integrated DELLA domains within putative plant DELLA proteins [70].

\section{Phylogenetic analysis of plant DELLA proteins}

Plant DELLA protein sequence alignment was performed using ClustalX software Version 2.1 [71] followed by the PHYLIP software package [72] to construct neighbor-joining (N) trees from the ClustalX alignment of the DELLAs. 1000 bootstrap replicates were generated to estimate support for the inferred relationships [73].

\section{Conserved motif analysis of plant DELLAs}

Conserved motifs in plant DELLAs were analyzed using the software MEME suite (version 4.11.1; http://meme. nbcr.net/meme/) [74]. Parameter settings: minimum motif width, 6; output motifs, 20; maximum motif width, 300 [73].

\section{Selective pressure analysis}

The ratios of non-synonymous to synonymous substitutions (i.e., $K_{\mathrm{a}} / K_{\mathrm{s}}$ or $\omega$ ) of gene groups were calculated using Codeml as implemented in PAML version 4.7 software [75].

\section{Codon usage bias analysis}

Parameters reflecting codon usage bias, including FOP, GC1, GC2, GC3s, GC12 content, RSCU, CAI, CBI, and exon length, were calculated using CodonW version 1.4.2 [76].

\section{Supplementary information}

Supplementary information accompanies this paper at https://doi.org/10 1186/s12870-020-02574-2.

Additional file 1: Table S1. Amino acid sequences of DELLAs in various species. Information includes the species name, gene name, amino acid sequence, and domain composition.

Additional file 2: Table S2. CDs sequences of DELLA NSGs and PSGs.

Additional file 3: Fig. S1. Conserved motifs of plant DELLAs.

Additional file 4: Fig. S2. Conserved motif sequences of plant DELLAs. The red box contains the conserved amino acid sequence of five-peptide "D-E-L-L-A" structure. The green box contains the conserved amino acid sequence of the "VHYNP" sequence.

\section{Abbreviations}

ABI: ABA INSENSITIVE; ARF: AUXIN RESPONSE FACTOR; BR: Brassinosteroid; PIF: PHYTOCHROME INTERACTING FACTOR; BZR1: BRASSINAZOLE-RESISTANT 1; EIN: ETHYLENE-INSENSITIVE; JAZ: JASMONATE-ZIM-DOMAIN PROTEIN; D14: DWARF 14; FLC: FLOWERING LOCUS C; RGL: RGALIKE; GAI: GA INSENS ITIVE; SLR1: SLENDER RICE 1; JA: Jasmonate; PSG: Positively selected gene; NSG: Negatively selected gene

Acknowledgments

Not applicable.

\section{Authors' contributions}

PW, FR, XW and HS designed the study. PW wrote the manuscript. PW, QZ, $Y C$ and $Y Z$ carried out data analysis. All authors have read and approved the manuscript, and ensure that this is the case.

\section{Funding}

This work was supported by the Major agricultural application technology innovation project of Shandong Province in 2018 "Research on product development and quality safety control of fresh grape extension industry chain", the Natural Science Foundation of Shandong province (ZR2019BC090), the National Natural Science Foundation of China (No. 31901866), the Agricultural scientific and technological innovation project of Shandong Academy of Agricultural Sciences (CXGC2016D01, CXGC2018E17, CXGC2018F04) and the Fruit innovation team of modern agricultural industry technology system in Shandong Province-Jinan comprehensive test station (SDAIT-06-21). The funders provided the funds but played no role in the study design, analysis of the data and in writing the manuscript.

\section{Availability of data and materials}

66 plant genomes and related data were downloaded from the Phytozome 12.1.6 database (https://phytozome.jgi.doe.gov/pz/portal.html), and the gymnosperm genomes and related data of Ginkgo biloba [68] was downloaded from GigaDB (http://gigadb.org/), Pinus pinaster was downloaded from SustainPine DB (http://www.scbi.uma.es/pindb/), and Picea abies was downloaded from the Congenie.org database (http://congenie. org).

Ethics approval and consent to participate Not applicable.

\section{Consent for publication}

Not applicable.

\section{Competing interests}

The authors declare that they have no competing interests.

\section{Author details}

${ }^{1}$ Shandong Academy of Grape, Shandong engineering research center for Grape cultivation and deep-processing, Jinan 250100, China. ${ }^{2}$ Key Laboratory of Urban Agriculture (East China), Ministry of Agriculture, Jinan 250100, China.

Received: 17 February 2020 Accepted: 23 July 2020

Published online: 06 August 2020

\section{References}

1. Richards DE, King KE, Ait-Ali T, Harberd NP. How gibberellin regulates plant growth and development: a molecular genetic analysis of gibberellin signaling. Annu Rev Plant Physiol Plant Mol Biol. 2001:52:67-88.

2. Olszewski N, Sun TP, Gubler F. Gibberellin signaling: biosynthesis, catabolism, and response pathways. Plant Cell (Suppl). 2002;14:S61-80.

3. Peng JR, Harberd NP. The role of GA-mediated signalling in the control of seed germination. Curr Opin Plant Biol. 2002;5:376-81.

4. Sun TP, Gubler F. Molecular mechanism of gibberellin signaling in plants. Annu Rev Plant Biol. 2004:55:197-223

5. Koornneef $\mathrm{M}$, van der Veen $\mathrm{JH}$. Induction and analysis of gibberellin sensitive mutants in Arabidopsis thaliana (L.) Heynh. Theor Appl Genet. 1980:58:257-63.

6. Wilson R, Heckman JW, Somerville C. Gibberellin is required for flowering in Arabidopsis thaliana under short days. Plant Physiol. 1992;100:403-8.

7. Sun TP, Kamiya Y. The Arabidopsis GA1 locus encodes the cyclase entkaurene synthetase a of gibberellin biosynthesis. Plant Cell. 1994;6:1509-18.

8. Murase K, Hirano Y, Sun TP, Hakoshima T. Gibberellin-induced DELLA recognition by the gibberellin receptor GID1. Nature. 2008;456:459-63.

9. Shimada A, Ueguchi-Tanaka M, Nakatsu T, Nakajima M, Naoe Y, Ohmiya H, et al. Structural basis for gibberellin recognition by its receptor GID1. Nature. 2008:456:520-3.

10. Pysh LD, Wysocka-Diller JW, Camilleri C, Bouchez D, Benfey PN. The GRAS gene family in Arabidopsis: sequence characterization and basic expression analysis of the SCARECROWLIKE genes. Plant J. 1999;18:111-9. 
11. Davière JM, Achard P. Gibberellin signaling in plants. Development. 2013; 140:1147-51.

12. Gao XH, Xiao SL, Yao QF, Wang YJ, Fu XD. An updated GA signaling 'relief of repression' regulatory model. Mol Plant. 2011;4:601-6.

13. Hou X, Lee LY, Xia K, Yan Y, Yu H. DELLAs modulate jasmonate signaling via competitive binding to JAZs. Dev Cell. 2010;19:884-94.

14. Li K, Gao Z, He H, Terzaghi W, Fan LM, Deng XW, et al. Arabidopsis DET1 represses photomorphogenesis in part by negatively regulating DELLA protein abundance in darkness. Mol Plant. 2015;8:622-30.

15. Li M, An F, Li W, Ma M, Feng Y, Zhang X, et al. DELLA proteins interact with FLC to repress the flowering transition. J Integr Plant Biol. 2016;58:642-55.

16. Lozano-Juste J, León J. Nitric oxide regulates DELLA content and PIF expression to promote photomorphogenesis in Arabidopsis. Plant Physiol. 2011;156:1410-23.

17. Marín-de la Rosa N, Pfeiffer A, Hill K, Locascio A, Bhalerao RP, et al. Wide Binding Site Analysis Reveals Transcriptional Coactivation of CytokininResponsive Genes by DELLA Proteins. PLoS Genet. 2015;11(7):e1005337.

18. Cheng H, Qin L, Lee S, Fu X, Richards DE, Cao D, et al. Gibberellin regulates Arabidopsis floral development via suppression of DELLA protein function. Development. 2004;131:1055-64.

19. Tyler L, Thomas SG, Hu J, Dill A, Alonso JM, Ecker JR, et al. Della proteins and gibberellin-regulated seed germination and floral development in Arabidopsis. Plant Physiol. 2004;135:1008-19.

20. Lee $\mathrm{S}$, Cheng H, King KE, Wang W, He Y, Hussain A, et al. Gibberellin regulates Arabidopsis seed germination via RGL2, a GAI/RGA-like gene whose expression is up-regulated following imbibition. Genes Dev. 2002;16:646-58.

21. Piskurewicz $U$, Lopez-Molina $L$. The $G A$-signaling repressor $R G L 3$ represses testa rupture in response to changes in GA and ABA levels. Plant Signal Behav. 2009:4:63-5.

22. Dill A, Sun T. Synergistic derepression of gibberellin signaling by removing RGA and GAl function in Arabidopsis thaliana. Genetics. 2001;159:777-85.

23. King KE, Moritz T, Harberd NP. Gibberellins are not required for normal stem growth in Arabidopsis thaliana in the absence of GAI and RGA. Genetics. 2001;159:767-76.

24. Lawit SJ, Wych HM, Xu D, Kundu S, Tomes DT. Maize DELLA proteins dwarf plant8 and dwarf plant9 as modulators of plant development. Plant Cell Physiol. 2010;51:1854-68.

25. Peng J, Richards DE, Hartley NM, Murphy GP, Devos KM, Flintham JE, et al. 'Green revolution' genes encode mutant gibberellin response modulators. Nature. 1999:400:256-61.

26. Ikeda A, Ueguchi-Tanaka M, Sonoda Y, Kitano H, Koshioka M, Futsuhara Y, et al. Slender rice, a constitutive gibberellin response mutant, is caused by a null mutation of the SLR1 gene, an ortholog of the height-regulating gene GAI/RGA/RHT/D8. Plant Cell. 2001;13:999-1010

27. Martí C, Orzáez D, Ellul P, Moreno V, Carbonell J, Granell A. Silencing of DELLA induces facultative parthenocarpy in tomato fruits. Plant J. 2007:52: 865-76.

28. Zhong GY, Yang Y. Characterization of grape gibberellin Insensitive1 mutant alleles in transgenic Arabidopsis. Transgenic Res. 2012;21:725-41.

29. Li X, Liu W, Li B, Liu G, Wei Y, He C, et al. Identification and functional analysis of cassava DELLA proteins in plant disease resistance against cassava bacterial blight. Plant Physiol Biochem. 2018;124:70-6.

30. Chen M, Maodzeka A, Zhou L, Ali E, Wang Z, Jiang L. Removal of DELLA repression promotes leaf senescence in Arabidopsis. Plant Sci. 2014;219220:26-34.

31. Fuentes S, Ljung K, Sorefan K, Alvey E, Harberd NP, Østergaard L. Fruit growth in Arabidopsis occurs via DELLA-dependent and DELLAindependent gibberellin responses. Plant Cell. 2012;24:3982-96.

32. Jin Y, Liu H, Luo D, Yu N, Dong W, Wang C, Zhang X, Dai H, Yang J, Wang E. DELLA proteins are common components of symbiotic rhizobial and mycorrhizal signaling pathways. Nat Commun. 2016;7:12433.

33. Yu N, Luo D, Zhang X, Liu J, Wang W, Jin Y, et al. A DELLA protein complex controls the arbuscular mycorrhizal symbiosis in plants. Cell Res. 2014;24:130-3.

34. Floss DS, Levy JG, Lévesque-Tremblay V, Pumplin N, Harrison MJ. DELLA proteins regulate arbuscule formation in arbuscular mycorrhizal symbiosis. PNAS. 2013;110(51):E5025-34

35. Achard P, Cheng H, De Grauwe L, Decat J, Schoutteten H, Moritz T, et al. Integration of plant responses to environmentally activated phytohormonal signals. Science. 2006;311:91-4.

36. Achard P, Gong F, Cheminant S, Alioua M, Hedden P, Genschik P. The coldinducible CBF1 factor-dependent signaling pathway modulates the accumulation of the growth-repressing DELLA proteins via its effect on gibberellin metabolism. Plant Cell. 2008;20:2117-29.

37. Achard P, Renou JP, Berthomé R, Harberd NP, Genschik P. Plant DELLAs restrain growth and promote survival of adversity by reducing the levels of reactive oxygen species. Curr Biol. 2008;18:656-60.

38. de Lucas M, Davière JM, Rodríguez-Falcón M, Pontin M, Iglesias-Pedraz JM, Lorrain S, et al. A molecular framework for light and gibberellin control of cell elongation. Nature. 2008;451:480-4.

39. Feng S, Martinez C, Gusmaroli G, Wang Y, Zhou J, Wang F, et al. Coordinated regulation of Arabidopsis thaliana development by light and gibberellins. Nature. 2008;451:475-9.

40. Cheminant S, Wild M, Bouvier F, Pelletier S, Renou JP, Erhardt M, et al. DELL As regulate chlorophyll and carotenoid biosynthesis to prevent photooxidative damage during seedling deetiolation in Arabidopsis. Plant Cell. 2011;23:1849-60.

41. Navarro L, Bari R, Achard P, Lisón P, Nemri A, Harberd NP, et al. DELLAs control plant immune responses by modulating the balance of jasmonic acid and salicylic acid signaling. Curr Biol. 2008;18:650-5.

42. Lan Z, Krosse S, Achard P, van Dam NM, Bede JC. DELLA proteins modulate Arabidopsis defences induced in response to caterpillar herbivory. J Exp Bot. 2014:65(2):571-83.

43. Cao D, Cheng H, Wu W, Soo HM, Peng J. Gibberellin mobilizes distinct DELLA-dependent Transcriptomes to regulate seed germination and floral development in Arabidopsis. Plant Physiol. 2006;142(2):509-25.

44. Gallego-Bartolomé J, Minguet EG, Marín JA, Prat S, Blázquez MA, Alabadí D. Transcriptional diversification and functional conservation between DELLA proteins in Arabidopsis. Mol Biol Evol. 2010;27(6):1247-56.

45. Vandenbussche F, Fierro AC, Wiedemann G, Reski R, Van Der Straeten D. Evolutionary conservation of plant gibberellin signalling pathway components. BMC Plant Biol. 2007;7:65.

46. Wang P, Song H, Li C, Li P, Li A, Guan H, et al. Genome-wide dissection of the heat shock transcription factor family genes in arachis. Front Plant Sci. 2017;8:106.

47. Itoh H, Sasaki A, Ueguchi-Tanaka M, Ishiyama K, Kobayashi M, Hasegawa Y, et al. Dissection of the phosphorylation of Rice DELLA protein, SLENDER RICE1. Plant Cell Physiol. 2005;46(8):1392-9.

48. Griffiths J, Murase K, Rieu I, Zentella R, Zhang ZL, Powers SJ, et al. Genetic characterization and functional analysis of the GID1 gibberellin receptors in Arabidopsis. Plant Cell. 2006;18(12):3399-414.

49. Cassani E, Bertolini E, Badone FC, Landoni M, Gavina D, Sirizzotti A, et al. Characterization of the first dominant dwarf maize mutant carrying a single amino acid insertion in the VHYNP domain of the dwarf8 gene. Mol Breeding. 2009;24:375-85.

50. Song H, Wang P, Li C, Han S, Lopez-Baltazar J, Zhang X, et al. Identification of lipoxygenase (LOX) genes from legumes and their responses in wild type and cultivated peanut upon Aspergillus flavus infection. Sci Rep. 2016;6:35245.

51. Yang $T$, Zhang L, Zhang $T$, Zhang $H, X u S, A n L$. Transcriptional regulation network of cold-responsive genes in higher plants. Plant Sci. 2005;169:987-95.

52. Zhang L, Vision TJ, Gaut BS. Patterns of nucleotide substitution among simultaneously duplicated gene pairs in Arabidopsis thaliana. Mol Biol Evol. 2002;19:1464-73.

53. Guo Y, Liu J, Zhang J, Liu S, Du J. Selective modes determine evolutionary rates, gene compactness and expression patterns in brassica. Plant J. 2017;91(1):34-44.

54. Vishnoi A, Kryazhimskiy S, Bazykin GA, Hannenhalli S, Plotkin JB. Young proteins experience more variable selection pressures than old proteins. Genome Res. 2010;20:1574-81.

55. Soskine M, Tawfik DS. Mutational effects and the evolution of new protein functions. Nat Rev Genet. 2010;11(8):572-82.

56. Wang P, Wang Y, Ren F. Genome-wide identification of the CLAVATA3/ EMBRYO SURROUNDING REGION (CLE) family in grape (Vitis vinifera L.). BMC Genomics. 2019;20(1):553.

57. Willige BC, Ghosh S, Nill C, Zourelidou M, Dohmann EM, Maier A, et al. The DELLA domain of GA INSENSITIVE mediates the interaction with the GA INSENSITIVE DWARF1A gibberellin receptor of Arabidopsis. Plant Cell. 2007; 19(4):1209-20.

58. Zhang B, Liu J, Yang ZE, Chen EY, Zhang CJ, Zhang XY, et al. Genome-wide analysis of GRAS transcription factor gene family in Gossypium hirsutum $L$. BMC Genomics. 2018;19(1):348.

59. Cheng S, Xian W, Fu Y, Marin B, Keller J, Wu T, et al. Genomes of subaerial Zygnematophyceae provide insights into land plant evolution. Cell. 2019; 179(5):1057-67. 
60. Wang P, Shi S, Ma J, Song H, Zhang Y, Gao C, et al. Global Methylome and gene expression analysis during early Peanut pod development. BMC Plant Biol. 2018;18(1):352.

61. Zhao H, Rossiter SJ, Teeling EC, Li C, Cotton JA, Zhang S. The evolution of color vision in nocturnal mammals. Proc Natl Acad Sci U S A. 2009;106(22): 8980-5.

62. Yu L, Wang XY, Jin W, Luan PT, Ting N, Zhang YP. Adaptive evolution of digestive RNASE1 genes in leaf-eating monkeys revisited: new insights from ten additional colobines. Mol Biol Evol. 2010;27:121-31.

63. Zhao H, Yang JR, Xu H, Zhang J. Pseudogenization of the umami taste receptor gene Tas $1 \mathrm{r} 1$ in the giant panda coincided with its dietary switch to bamboo. Mol Biol Evol. 2010;27:2669-73.

64. Song H, Sun J, Yang G. Comparative analysis of selection mode reveals different evolutionary rate and expression pattern in Arachis duranensis and Arachis ipaënsis duplicated genes. Plant Mol Biol. 2018:98(4-5):349-61.

65. Li Z, Baniaga AE, Sessa EB, Scascitelli M, Graham SW, Rieseberg LH, et al. Early genome duplications in conifers and other seed plants. Sci Adv. 2015; 1(10):e1501084.

66. Chen SL, Lee W, Hottes AK, Shapiro L, McAdams HH. Codon usage between genomes is constrained by genome- wide mutational processes. Proc Natl Acad Sci U S A. 2004;101(10):3480-5.

67. Knight RD, Freeland SJ, Landweber LF. A simplemodel based on mutation and selection explains trends in codon and amino- acid usage and GC composition within and across genomes. Genome Biol. 2001;2(4): research0010-research0010.0013.

68. Guan R, Zhao Y, Zhang H, Fan G, Liu X, Zhou W, et al. Draft genome of the living fossil Ginkgo biloba. Gigascience. 2016;5(1):49.

69. Finn RD, Clements J, Eddy SR. HMMER web server: interactive sequence similarity searching. Nucleic Acids Res. 2011;39:W29-37.

70. Letunic, et al. SMART: recent updates, new developments and status in 2015. Nucleic Acids Res. 2015;43(Database issue):D257-60.

71. Larkin MA, Blackshields G, Brown NP, Chenna R, McGettigan PA, McWilliam H, et al. Clustal W and Clustal X version 2.0. Bioinformatics. 2007:23:2947-8.

72. Baum BR. PHYLIP: phylogeny inference package. Version 3.2. Q Rev Biol. 1989:64:539-41.

73. Wang P, Su L, Gao H, Jiang X, Wu X, Li Y, et al. Genome-wide characterization of bHLH genes in grape and analysis of their potential relevance to abiotic stress tolerance and secondary metabolite biosynthesis. Front Plant Sci. 2018;9:64.

74. Bailey TL, Williams N, Misleh C, Li WW. MEME: discovering and analyzing DNA and protein sequence motifs. Nucleic Acids Res. 2006;34(suppl 2): W369-73.

75. Yang Z. PAML 4: phylogenetic analysis by maximum likelihood. Mol Biol Evol. 2007:24:1586-91.

76. Peden JF. Analysis of codon usage. UK: PhD Thesis, University of Nottingham; 1999

\section{Publisher's Note}

Springer Nature remains neutral with regard to jurisdictional claims in published maps and institutional affiliations.

Ready to submit your research? Choose BMC and benefit from:

- fast, convenient online submission

- thorough peer review by experienced researchers in your field

- rapid publication on acceptance

- support for research data, including large and complex data types

- gold Open Access which fosters wider collaboration and increased citations

- maximum visibility for your research: over $100 \mathrm{M}$ website views per year

At $\mathrm{BMC}$, research is always in progress.

Learn more biomedcentral.com/submissions 